\title{
Anatomía vegetal en San Alberto Magno
}

\author{
JOSÉ MARÍA VALDERAS
}

\begin{abstract}
VALDERAS, J. M. (1987). Vegetal anatomy in Albertus Magnus. Collect. Bot. (Barcelona) 17(1): $125-134$

Since T. A. Sprague wrote his outstanding essays many years ago on the meaning of botanical terms used by Plinius, Isidorus, Albertus and Ruellius, no one else had resumed this task, which is essential for the history of botany.

Though Albertus explained vegetal structure in terms of the function it fulfils, the methodology used here ignores Physiology and concentrates on Morphology. After discussing the composition of De Vegetabilibus, we deal with organs using the order and division established by Albertus: succus, nodus, radix, venae, stipes, ramus, cortex, flos, folium, fructus, and so on.
\end{abstract}

Keywords: Morphology, Albertus Magnus, History of Botany.

\section{Resumen}

Valderas, J. M. (1987). Anatomía vegetal en San Alberto Magno. Collect. Bot. (Barcelona) $17(1): 125-134$

Desde los ya lejanos, y meritísimos, trabajos de T. A. Sprague sobre el significado de los términos botánicos empleados por Plinio, Isidoro, Alberto y Ruellius, nadie había reemprendido tan necesaria tarea para la historia de la botánica.

Aunque la razón de una estructura vegetal se explica, en San Alberto, por la función que cumple, aquí prescindimos metodológicamente de la fisiologia para ceñirnos a la morfología. Tras exponer la composición del De Vegetabilibus, abordamos los distintos órganos de la planta según el orden y la división trazados por él: succus, nodus, radix, venae, stipes, ramus, cortex, flos, fructus, etcétera.

Palabras clave: Morfología, San Alberto Magno, Historia de la Botánica.

\section{Introducción}

La historiografia relativa a la obra botánica de San Alberto (finales del XII-1280) arranca con el "descubrimiento" del valor extraordinario de la misma por parte de Ernst Meyer, quien

J. M. VAlderas: Institut Botànic de Barcelona. Avinguda dels Muntanyans s/n. Parc de Montjuïc. 08004 Barcelona. 
la compara con la de Teofrasto, Gesner y Cesalpino (1), toma nuevo aliento con sendos artículos de T. A. Sprague (2) y entra en un nuevo enfoque más crítico con los trabajos aparecidos con motivo del séptimo centenario de su muerte (3).

La obra botánica de San Alberto se compendia en De vegetabilibus et plantis libri septem (4), escritos en la segunda mitad del siglo XIII, antes de que ocupara la sede episcopal de Regensburg (marzo de 1260). Los cinco libros primeros son predominantemente teóricos y de aplicación práctica los dos últimos, dedicando el sexto a la terapéutica y discernimiento de las distintas especies y el séptimo a la agricultura y jardinería. Los libros primero y cuarto glosan, con interpolaciones propias, el De plantis de Nicolás de Damasco, supuestamente atribuido a Aristóteles (5); el segundo, tercero y quinto son digresiones personales.

Las fuentes en que se apoya son el texto del Pseudoaristóteles citado, Dioscórides, Plinio e Isidoro, los herbolarios medievales en todas sus variantes, el pensamiento árabe (Avicena sobre todo) y, por encima de cualquier autoridad, la experiencia propia. Entendida en sentido lato: la praxis agrícola, la observación recogida en sus frecuentes viajes por Centroeuropa y los experimentos que acometió (con injertos, por ejemplo); empirismo que constituirá el criterio definitivo de sus trabajos naturalistas (6).

\section{La ciencia de la botánica}

Para San Alberto había una ciencia general de la vida, que había desarrollado en el $D e$ anima, cuyo campo semántico abarcaría el conjunto de propiedades que caracterizan a un organismo, y había tres ciencias subalternas de la anterior que abordaban, respectivamente, las propiedades específicas de plantas, animales y hombre (7). La botánica, la zoología y la antropología eran, las tres, ciencias biológicas, pero no lo eran por igual. Las propiedades del ser vivo le convenían más al hombre que al animal y a éste más que a la planta. Los tres son seres vivos, pero analógicamente, es decir, y sin entrar en pormenores, subordinadamente (8). Ese es el motivo por el que, al explicar órganos y funciones de los vegetales, acote su paralelismo o diferencia con respecto a los animales.

La vegetabilidad o razón de ser de la planta le viene del alma, principio unificador y origen de las tres funciones características: nutrición, desarrollo y propagación (9). El alma ejerce sus funciones a través del cuerpo vegetal, es decir, de sus órganos: raíces, tallo, ramas, frutos, hojas, etcétera (10). Y aquí conviene introducir, de nuevo, el concepto de analogía. Todos los seres carentes de movimiento local, por supuesto vivos, y de crecimiento casi ilimitado son plantas, pero no todas lo son por igual. Reconoce cinco grandes grupos: arbores, frutices, olera, herbae et fungi (11), ordenados de mayor a menor perfección vegetal; la perfección depende del número de partes poseídas, de la forma de reproducción y de caracteres que hoy nos parecen secundarios (la dureza, por ejemplo).

\section{Anatomía}

$\mathrm{Al}$ abordar la anatomía, distingue entre partes integrantes o constitutivas y partes accidentales (12). De las primeras, algunas son potenciales, como el succus, "que es el humor atraído por las raíces, a través de sus poros, para alimentar todas las partes de la planta distribuido mediante la virtud nutritiva" (13). Este constituyente se llama potencial porque se recibe indeterminado en la raíz e insípido. A medida que va ascendiendo desde ésta, va adquiriendo las propiedades - "sabores"- de los órganos que atraviesa y se conforma a la manera de ser de la planta; en esas transformaciones interviene el calor interno necesario (14). El succus no digerido se corrompe y sale al exterior en forma de epífitos y excrecencias (15).

Otras partes integrantes son actuales. Se dividen éstas, a su vez, en otros dos grupos en razón de la nutrición: órganos que cumplen una misión ministerial del transporte de la nutrición y otros que sirven de reservorio del jugo nutricio y de lugar de mayor elaboración, o 
digestión, del mismo (16). Siguiendo su propia exposición, empezaremos por los segundos: los nudos y, en particular, el malleolus (17).

\section{Nudos}

Las plantas que presentan nudos suelen caracterizarse por su riqueza en medula o son, por contra, enteramente huecas y muy largas; y cita como ejemplos Vitis, Clematis, Triticum, Arundo, Avena y algunos más. Son éstas especies consistentes ("rarae substantiae"), que se oponen a las carentes de nudos ("debilis substantiae"), verbigracia, los juncos (18). El número de nudos es proporcional a la longitud de la planta, como observamos en Polygonum, Valeriana, Foeniculum, etc. En otros casos, así en las gramíneas, raramente sobrepasa de cuatro el número de nudos (19). Número cuaternario que está, además, en consonancia con la cifra de "digestiones" cabales, la última de las cuales tiene por función transformar el jugo nutricio en substancia semejante a la de la especie en que se halla (20). La disposición de los nudos no guarda una distancia constante en el culmo. El primero y más cercano al suelo suele ser más negruzco y posee más del elemento tierra. (Recuérdese que, para los medievales, los elementos componentes de todas las partes orgánicas eran cuatro: tierra, agua, aire y fuego, con distinto predominio de uno u otro según el órgano en cuestión.) El segundo nudo dista del primero más que éste de la tierra y posee más elemento acuoso también, por cuya razón echa ya hojas. Del segundo al tercero comienza a estrecharse la caña en las gramíneas, y más todavía del tercero al cuarto, debido ello a la mayor digestión del material nutricio (21).

Expuesta la parte integrante actual más importante -el nudo-, describe las estructuras que pueden asimilársele; por ejemplo, los puntos de inserción de las ramificaciones secundarias del tallo, que se hacen especialmente patentes en la hiedra y en ciertas plantas trepadoras o reptantes (22). A modo de colofón de este apartado, señala que, debido a su función reservante y purificadora, de los nudos salen las hojas, en cuya formación se aprovecha lo residual de la depuración del juego nutricio y cuya misión es proteger a la planta.

\section{Raíz}

En el transporte del alimento desempeña un papel capital la raíz. La planta tienen en ésta su boca (23). Y su corazón, en el sentido de que, una vez sumido por atracción el alimento del suelo, le infunde calor vivificante para que pueda alcanzar los demás órganos. Se trata, por supuesto, de dos expresiones metafóricas: la raíz hace las veces de boca y ocupa el corazón de la planta (24). No le atribuye, empero, ninguna de las funciones del estómago -que el galenismo cifraba en la separación del elemento térreo del fluido nutriente puro- porque tal "digestión" se producía en el suelo, ni del hígado -donde se filtraba, así se creía, la masa nutriente pura de la acuosidad impura o superflua (25). Las raíces absorbían ya el alimento bastante puro a través de los meatos radicales. Distingue entre plantas con raíces duras y plantas con raíces blandas (26).

\section{Venas}

Admite la existencia de un sistema vascular que vehicula el transporte del jugo nutricio y el seminal. Para el primero prefiere el término vena, para el segundo el de vas. Las venas son vasos ascendentes que portan la nutrición, y no tienen nada que ver con las homónimas de los animales, apostilla (27). Gracias a ellas, las plantas crecen por superposición de una túnica sobre otra, sean herbáceas o leñosas. Unas veces, esos vasos son tortuosos, y tenemos las plantas nudosas. Otras, se disponen en red: unos verticales y otros horizontales (28).

Las venas arrancan, unas, de la raíz; otras, de la medula. Aquéllas siguen un curso ascendente; éstas van del centro al exterior, como los radios de una circunferencia (29). Estas viae nutrimenti, venae o viae venales poseen, además, la consistencia necesaria para poder transportar el jugo nutricio (30). Carecen, empero, de pulsatilidad, es decir, de la fuerza impulsora, que se halla ínsita en el propio alimento. 


\section{Tallos y troncos}

Tras el estudio de nudos, raíz y venas, acomete la descripción del tallo o tronco. No emplea todavía la distinción entre caulis y caudex, sino que prefiere, por encima de cualquier otro, el término stipes. Si es de cierto porte, usará a veces truncus; si de arbusto o mata, crus. Del tronco brotan ramificaciones de distinto orden: ramus, ramusculus, virga flagra (31). La región más interna del tallo está formada por la medula (medulla). La entiende como una prolongación de la raíz, su vicaria (32). Se trata de una vía de transporte nutricio y seminal. Gracias a ella, arriba a las partes de la planta el sustento necesario. Desde la medula se propagan horizontalmente los canalículos que han de alimentar, a lo ancho, el tronco. En sus comienzos es blanca, pero andando el tiempo se torna anaranjada (33). E igual que hiciera con las raíces, divide los vegetales en razón de su medula: los hay con mucha y otros con poca. Ejemplo de los primeros es el Sambucus. Bastante rica en ella es la Vitis; en cambio, totalmente huecas son las cañas (34).

La corteza, cortex, es la capa de recubrimiento y protección de la planta. Es a los vegetales lo que el pellejo a los animales, aunque no está tan pegada al "cuerpo" (35). Y así como en éstos la parte desollada no crece, sino que cicatriza, también en aquéllos observamos idéntico fenómeno, por cuya razón acaban muriendo las plantas decorticadas (36). Pero la formación de uno (pellejo) difiere de la formación de la otra (corteza): la piel de los animales nace de la imbricación de las venas, en tanto que la corteza es un producto de la digestión, que no ha podido depurar el "humor" completamente y llega a la superficie; de ahí su consistencia escamosa y térrea. Por lo mismo, falta de ductilidad, se cuartea. Unas veces lo hace radialmente (Prunus); otras, verticalmente (Quercus). Esto último se aplica, con propiedad, a la corteza externa, áspera y dura. La corteza interna es más blanda (37). Entre la medula y la corteza interna distingue el lignum, blanco (38). Llama tunicas ligneae a los estratos delgados y concéntricos que corresponden a períodos de desarrollo del xilema, es decir, a los anillos anuales de las plantas leñosas, sin olvidarse de la forma de crecimiento peculiar de la palmera (Phoenix dactylifera) (39).

\section{Hojas}

Hoja era tanto la simple como los foliolos de la compuesta. Folium significaba también lo que hoy entendemos por brácteas, sépalos y pétalos. El raquis era el ramus folialis, la virgula parvula, la linea longa e incluso el genérico stipes. Este último nos lleva, por asociación semántica, a la denominación del pecíolo (crus), llamado también cotyledo (40). Las hojas que nacen del tallo, pensemos en las matas, arrancan directamente de los nudos. Deben su formación, igual que la corteza, a la "superfluidad" resultante de la digestión del jugo nutricio. Y sirven también para proteger a la planta de las lesiones y las inclemencias del sol (41). Esa misión defensiva la cumplen, sobre todo, con los frutos, de los que difieren en materia, figura y forma (42). La linea recta o raquis divide la hoja en dos arcos simétricos la mayoría de las veces; la venación sirve para hacer llegar el sustento a toda la lámina foliar (43). Distínguense por la forma del limbo, tipo de venación, tamaño y espesor (44).

La morfología actual distingue entre espinas y aguijones, entre otras razones porque aquéllas están lignificadas y poseen tejido vascular, en tanto que los acúleos son de origen superficial. La agudeza de observación resulta, en este caso, asombrosa: "Encontramos dos géneros de espinas en las plantas. Làs hay que nacen de lo más hondo, son rectas y largas... Pero hay otras que no se ahondan en el cuerpo de la planta, sino que se adhieren en la corteza externa y son más cortas, recorvadas y afiladas" (45). Asocia a éstos los tricomas o pelos urticantes, pili (46).

Venas, medula, corteza y lignum constituyen las partes simples de la planta. De éstas, o de algunas, se forman las compuestas: raíces, tallos, ramas, frutos, hojas, así como las subdivisiones de éstas. Compete al tronco servir de soporte del vegetal; a las raíces, aportar el 
alimento del individuo y asegurar la pervivencia de la especie. Ni ramas ni ramitas se ordenan a ninguna misión relativa al individuo, sino que propio de ellas es florecer y fructificar para perpetuar la especie (47). De ello se sigue que todas las partes compuestas de la planta hallan en la raíz su progenitor y su administrador, de quien reciben la sustancia nutricia y el vigor, substancia que las demás partes convierten en riqueza para los descendientes (48).

\section{Flor y fruto}

Las partes ordenadas a la propagación de la especie vegetal son la flor y el fruto. La flor es el anuncio del fruto. Pero, por razón de su naturaleza material, se relaciona también con la hoja (49). Comparte con el fruto la substancia, y ello explica que se den juntos, siendo unas veces la flor súpera y otras ínfera (50). Aparece por encima del fruto en Pyrus, Malus, Punica, y, por debajo, o ciñendo al fruto, en Papaver, Amygdalus. La flor súpera suele darse en los árboles, aunque no en todos; la ínfera, en las "herbae". Matiza, empero, que no es esa ninguna regla general (51). Cita lo que en su tiempo, y desde mucho antes, se creía que era excepción: la higuera que, igual que ciertas rosáceas, poseerían fruto sin flor previa (52). Él añade un razonamiento justificador; la higuera, en virtud de su humedad viscosa, impediría el decantamiento del elemento acuoso formador de las flores; la excepción de las rosáceas, muy infrecuente, deberíase a la situación opuesta, esto es, a la pérdida de la humedad antes de cristalizar en flor (53).

Además del lugar que ocupa la flor con respecto al fruto, le interesan a San Alberto otras propiedades. La figura, por ejemplo. Esta varía cualitativa y cuantitativamente. Pero destaca tres morfologías generales: aviforme, campaniforme (y piramidiforme) y asteriforme, con la combinación también habitual de las formas en campana y en estrella. (54). Ofrece un aspecto aviforme la "aquilea" (Aquilegia, aguileña). Esta planta tiene flores de cinco pétalos prolongados en la base en forma de espolón, no cuatro como escribe nuestro autor. Ve esa misma forma de ave en las corolas zigomorfas de la ortiga muerta (Lamium album) y la violeta (Viola), esta última también con espolón según es notorio. Flor acampanada (o piramidal) es, dice, la de muchas plantas, por ejemplo, la del aligustre (Ligustrum), con corola embudada, y el helenio (Inula helenium) (55). Por asteriforme entiende la figura que dibujan las flores que, como Rosa, se abren en radios. Retazos de campaniforme y asteriforme ofrecen Lilium, Agrostemma y Punica (56). La razón que aduce para explicar esas figuras estriba en la constitución material de los distintos tipos de flor: la aviforme sería muy viscosa, poco la asteriforme y a medio camino se encontraría la campaniforme (57).

No mantiene una distinción tajante entre flor e inflorescencia. El ramillete floral del orégano (Origanum vulgare) o el ramillete de ramilletes del yezgo (Sambucus) son una sola flor, pero en ambos casos señala que se disponen en "corona" (58). Idéntica apreciación le merecen muchas labiadas y compuestas. Sería, por lo demás, pretencioso o fuera de lugar esperar que discriminara entre corimbos, umbelas, capítulos y otras inflorescencias, simples o compuestas sin conocer la función reproductora de la flor. Pero sí da nombre a ciertas inflorescencias características: spica de las gramíneas y botrys al tirso de Vitis.

\section{Partes de la flor}

¿Qué entendía por flor? Comencemos por recordar lo que no era: los sépalos o brácteas (59). El resto de la noción moderna entraba con mayor o menor precisión. Cierto es que a veces toma la parte por el todo, como en el caso de la manzanilla (Matricaria chamomilla) en donde, por otro lado, no hace más que repetir un texto atribuido a Dioscórides posteriormente. Si en la manzanilla eran los pétalos quienes asumían la noción de flor, en Clematis vitalba y en Vitis vinifera eran los estambres los que constituían la flor. Y en este caso también, más que una restricción es una defensa contra quienes rechazaban la existencia de flor en la vid (60). Los pétalos, por otro lado, siempre se refieren como folia floris, las hojas de la flor. Otros 
elementos son las spicae o lineae, traducibles por filamentos o estambres; intercambiables éstos, a su vez, con los de virgula (estilo), nodum (estigmas o anteras). Aunque no separa los órganos masculinos de los femeninos, sí evidencia una curiosa exactitud en los detalles. Así, a propósito de Lilium candidum, la azucena, cuyas flores, muy grandes, forman un ramillete terminal y están constituidas por seis piezas, y cuyos estambres en número de seis también presentan los filamentos blancos y las anteras prolongadas, oscilantes y de un amarillo intenso (61).

Tal vez la descripción más cabal de la flor nos la ofrezca al detallar la morfología de la borraja (Borago officinalis). Se trata de una "herba" de hojas ásperas y tallo lanuginoso, con flores asteriformes, compuesta de cinco círculos, integrados, a su vez, por cinco piezas: de fuera adentro, theca floris (cáliz), verde; folia floris (lóbulos de la corola), semejantes al jacinto; quinque parvulae eminentiae in flore ipso (segmentos de la corona), como el jacinto por arriba y blancos por abajo; en cuarto lugar, y a medida que avanzamos intus floris-expresión que nos acerca a la noción albertiana de flor muy próxima a la nuestra-, encontramos quinque virgulae (los estambres), rectos, y en medio de ellos, por último, una virgula (el estilo), más larga que los estambres. Ambos, estilos y estambres, constituyen las espigas de la flor (spicae floris). (62).

Dentro de la flor, el intus floris al que aludiámos antes, la inmensa mayoría de las plantas (omnis fere flos) tiene "granos" (estigmas o anteras), prendidos en unos pedículos (coctiledones) sujetos al fondo de la concavidad floral. El número de esos elementos varía con las plantas (63). Confunde, sin embargo, el polen de las anteras con el néctar, movido a error quizás por la libación de las abejas.

Los colores de las flores, como el aroma, dimanan de su constitución elemental o material. En el interior, el color del polen es amarillo (croceum formale). No se da, salvo rarísimas excepciones, si es que hay alguna, ni el verde ni el negro. Los más abundantes: blanco, azul, rojo, jacinto y púrpura (64).

En los verticilos florales observa la disposición alternante de pétalos exteriores e interiores y de sépalos del cáliz (theca o siliqua, dos términos de amplia significación en la botánica albertiana, pero con la idea siempre de cubrir o cerrar algo). Se trata de un fenómeno habitual que tiene una función de defensa, a modo de distintos obstáculos a saltar por el agresor (65). En esa misma línea de explicación racional de la anatomía de la flor podríamos agregar la justificación mecánica de la dilatación y constricción ante el cambio de temperatura del día y la noche. No se trata de ningún movimiento -característica de los animales-, sino de que el calor dilata los órganos florales y el frío los encoge, al hacerlo con los constituyentes elementales de esos órganos (66).

Por último, la concavidad del aparato floral. Formada por el elemento ventoso evaporado del constituyente húmedo sutil de la materia floral, facilitará la producción del néctar y del polen que recogerán abejas y otros animales pequeños (67). Ese fluido húmedo y ventoso, por decirlo con sus palabras, constituirá el primordio ovárico. Cuando a la flor le falta la alimentación sutil que constituye su materia, cae (68). También la hoja cae cuando muere. No ocurre lo mismo con el fruto, que no cumple plenamente su cometido hasta que no se desprende del pie en que se formó (69).

\section{Partes del fruto}

En la carpología, San Alberto sigue la misma pauta que se ha marcado en los demás órganos: su estudio científico o en general, sin descender a los importunos detalles de los casos particulares (70). Y ello comporta ahondar en su naturaleza: "vapor autem siccus ventosus est materia fructus: propter quod etiam fructus secundum genus suum ventosi esse judicantur." (71).

La definición nominal del fruto no nos revela su constitución: fruto es lo que se disfruta. 
Secundum autem rem, es decir, de acuerdo con su naturaleza o lo que la naturaleza ha querido que sea, fruto es, primordialmente, la semilla a través de la cual pervive y se propaga la especie, en razón del poder generador que lleva ínsita en sí misma (72). Por cuyo motivo hasta los frutos no destinados a la fruición de los mismos, como los agrios y venenosos, son auténticos frutos, pues sirven para la conservación y multiplicación de la especie.

$\mathrm{El}$ fruto consta de caro y nucleus. La carne recibe su substancia de las digestiones realizadas en el tronco y las ramas; el núcleo, de la raíz (73). La razón estriba en que la virtud encerrada en la raíz informa o da sentido a toda la planta. Ambos fluidos llegan al punto de gemación (locum gemmae), suben por el pedicelo del fruto (coctilido) y se separan: uno, nutricio, formará el fruto; el otro, germinal, la semilla (semen). La caro ayudará, con su calor, a la maduración de la semilla. En ocasiones, una película, más o menos dura o coriácea, dividirá ambas partes: la testa.

El pericarpio (acepción más frecuente de fructus) presenta distintas texturas que compete al botánico (physicus) discernir. Unas plantas lo tienen laxo, duro y compacto otras, blando éstas, pulposo aquéllas, etcétera (74). El proceso de endurecimiento de la testa y de la caro es un proceso digestivo, que corre paralelo al de la maduración de la semilla encerrada.

La semilla (semen, nucleus) constituye la planta en potencia. Tiene en su ser el sujeto de la virtud formativa (75). Que, en un comienzo, recurre para su labor germinal a la sustancia reservante que le rodea (farina, residuum substantiae seminis). Y así como el fluido seminal de los animales es viscoso, también el de la semilla es oleaginoso y grasiento (76).

La testa nace de la excrecencia de la combustión o digestión de las semillas; por ser ese residuo "terrestre" es natural que sea coriáceo y negro a veces (77). Aduce el ejemplo de la peonía (Paeonia officinalis); ésta, como es sabido, tiene el fruto con numerosas semillas redondas, de primero rojas y luego negras. Muchas especies carecen de involucro carnoso y se limitan a protegerse con una lámina (siliqua), o vaina. Tampoco poseen "carne" los frutos de las gramíneas, cuyas semillas maduran en glumas (palea) (78).

Una vez madurada la semilla, gracias al hervor mantenido por el pericarpio duro que impide la evaporación de la humedad radical, el fruto se abre o se desprende, a veces con ímpetu, del pie (79). Ese desarrollo seminal corre paralelo al del pericarpio (fructus pepanus), cuyo signo exterior es la coloración que se torna apetitosa para el hombre o para los animales (80). El proceso de maduración del fruto atraviesa cuatro fases: la de formación con el jugo nutricio de la planta; la de crecimiento; la de perfeccionamiento, es decir, cuando adquiere el tamaño idóneo y cesa de atraer el jugo nutricio, y, por último, el de madurez, cuando el calor termina con la "humedad" retenida (81).

La figura es un carácter discriminante que San Alberto estudia en los distintos órganos que analiza. También en los frutos y las semillas. Figura que responde a "rationes physicas" (82). Dos son las principales: esféricas y columnares. Sabido es que la esfera era la figura geométrica privilegiada en el mundo medieval, ya que desde el centro se irradiaba a todos los puntos con igual fuerza; la propia figura columnar, escribe nuestro autor, "generatur ex circulo sursum super lineam rectam moto" (83). E incluso de la circular dimana la piramidal, en su opinión. Columnar era el trigo, por ejemplo; esférica Vicia faba; piramidal, Hordeum. Entre los frutos se acerca a la esfericidad Malus; Pyrus es piramidal, columnar Cucumis. Los hay trigonales y cuadrados, cuya explicación no debe buscarse fuera de su propia naturaleza (84).

Además de por su figura, los frutos se distinguen por su relación con la semilla. Considerará, así, tres grandes clases: frutos con semillas protegidas con una siliqua o pellicula, frutos cuyas semillas se hallan insertas en otras sustancias envolventes, sumamente variables a su vez, y semillas casi desnudas. Ejemplos de las primeras podrían ser las cápsulas de Papaver y Reseda. (No se pierda de vista que la terminología no está fijada y que, por ende, una misma estructura recibe distintos nombres y un mismo nombre se aplica a distintas estructuras, aunadas eso sí por idéntica función. Para nuestra carpología convencional, por ejemplo, cápsula es el fruto de Papaver, Agrostemma, Viola e Hyoscyamus; para san Alberto, el fruto de 
la amapola era una siliqua o un caput, el de la neguilla un vas seminis, un folliculus el de la violeta y una theca el del beleño.)

Vayamos con la segunda clase de frutos: aquellos cuyas semillas se encuentran insertas en substancias envolventes. (85). Se engloban aquí pomos y hesperidios, que encierran las semillas en lóculos (camerae). Drupas y bayas que, además, poseen os (Prunus, Phoenix). Frutos secos y dehiscentes se encuadran también aquí, como las legumbres (Cicer, Vicia, Pisum, etcétera), susceptibles a veces de ser arrojadas muy lejos (86). Esta clase de frutos suele caracterizarse por presentar tres involucros (cooperturae): la corteza (cortex) o epicarpio, el hueso y la película tenuísima que recubre la semilla propiamente dicha (la testa que llamaríamos hoy). Testa, sensu albertiano, es el pericarpio duro de los frutos secos y dehiscentes (87).

La última clase de frutos la integran las semillas desnudas. Se adscriben en este tercer grupo los granos de las gramíneas dotadas de culmo herbáceo y algunas más (88).

La anatomía vegetal conduce a la sistemática, pues es la posesión de los órganos y caracteres lo que permite adscribir al individuo vegetal a una especie y ésta a un grupo. La anatomía constituye, asimismo, el substrato de la fisiología, de las "operaciones". Dos capítulos muy importantes de la botánica albertina que, sin embargo, se escapan ahora de nuestro propósito.

\section{NOTAS}

(1) MeYER, Ernst: “Albertus Magnus. Ein Beitrag zur Geschichte der Botanik im dreizehnten Jahrhundert”, Linnaea 10 (1836), 641-741. Idem: "Albertus Magnus, Zweiter Beitrag zur erneuerten Kenntniss seiner botanischen Leistungen”, Linnaea 11 (1837), 545-595. MEYER, Ernst et Carl JesSEn (eds.): Albertus Magnus: De Vegetabilibus libri septem; Berlin, Georg Reimer, 1867.

(2) Sprague, T. A.: "Plant Morphology in Albertus Magnus", Kew Bulletin 9 (1933), 431-440. Idem: "Botanical Terms in Albertus Magnus", Kew Bulletin 9 (1933), 440-459.

(3) Albertus Magnus. Doctor Universalis, Mainz, 1980. Y, sobre todo, Albertus Magnus and the Sciences. Commemorative Essays, 1980. Edited by James A. Weisheilp, O. P.; Pontifical Institute of Mediaeval Studies; Toronto, 1980. En este volumen se acomete el estudio de los seis primeros libros, de los siete que contiene, del De Vegetabilibus. Karen Reeds aborda los cinco primeros en "Albert on the Natural Philosophy of Plant Life" (341-354) y Jerry Stannard, el sexto: "Albertus Magnus and the Medieval Herbalism" (355-377).

(4) Aquí manejaremos la edición de Augustus Borgnet: Beati Alberti Magni Ratisbonensi Episcopi, Ordinis Praedicatorum, Opera omnia. Parva naturalia. De Vegetabilibus et plantis; Parisiis, Apud Ludovicum Vivès; MDCCCXCI.

(5) Sobre la historia de la parternidad del De plantis, véase M. Bouyges: "Sur le De plantis d'Aristote-Nicholas à propos d'un manuscript arabe de Constantinople”, Mélanges de l'Université Saint-Joseph, Beyrouth 9 (1924), 71-89. B. Hemmerdinger: "Le De plantis, de Nicholas de Damas à Planude", Philologus 111 (1967), 56-65, entre otros. Con gran acierto, The Complete Works of Aristotle. The Revised Oxford Translation (Princeton University Press, 1984) incorpora el texto del De plantis, traducido por E. S. Forster.

(6) Comm. in II Sentent. 13, C. art. 2. Opera omnia. Ed. Borgnet, vol. $27,247$.

(7) De vegetabilibus et plantis, ed. cit., I, i, 1.

(8) De veg. I, i, 2.

(9) $I d$.

(10) Id.

(11) De veg. V, i, 2.

(12) Id. II, i, 3 .

(13) "Est autem succus humor qui per poros radicis attractus, ad nutriendam totam plantam per plantae partes a virtute nutritiva distribuitur" (Id.).

(14) Id.

(15) $I d$.

(16) Id.

(17) "Partes autem quae actu sunt partes plantae, dividuntur in duo genera: quaedam enim sunt sicut membra officialia ad nutrimenti officium deserventia: sicut lignum in habentibus lignum, vel caro herbalis in non habentibus lignum, est sicut membrum simile in animalibus, cortex autem sicut pellis in animalibus: et ad hunc modum est de caeteris partibus plantae. Nodi enim licet triplices invenuntur in plantis: tamen nodus ille qui vocatur malleolus, est factus a

(18) Id. natura ut in ipso stet succus, et ampliorem accipiat digestionem" (Id.).

(19) Id.

(20) Id.

(21) Id. 
(22) "Sunt autem quaedam plantae aliquid similes nodi habentes, quod tamen, verissime nodus non est, sicut hedera, et quaedam herbae aquatices longas, quae radices habent acutas et parvas quas infigunt lapidibus et lignis, super quae (23) Id.

(24) Id.

(25) $I d$.

(26) $I d$.

(27) "Vena autem in planta proprie loquendo non est, sed neque secundum multum manifestum similitudinem, sed viae nutrimenti dicuntur venae ejus, quae aliquando sunt directe ascendentes, et nunc crescit planta quasi per quasdam tunicas herbales vel ligneae" (Id.; cfrt. et. III, i, 5).

(28) Id.

(29) De veg. IV, iii, 5.

(30) De veg. II, i, 3 .

(31) Id. Cf. etiam V, i, 2; V, i, 7, etc.

(32) De veg. II, i, 3; V, 1, 5 .

(33) De veg. II, i, 3.

(34) "Sunt autem plantae quaedam fere totam substantiam habentes medullosam, sicut sambucus, et ebulus, et hujusmodi: et omnes illae nodos habent multos, et nutriuntur ex medulla: et ideo est multa in eis: et in hoc genere est vitis, sed minus est medullosa quam sambucus et ebulus. Alias autem experimur plantas in toto concavas sicut

(35) Id. fistulas quasdam et calamos." (Id.).

(36) Id. Cf. etiam III, i, 2; III, i, 5; V, i, 2.

(37) De veg. II, i, 3; V, i, 2.

(38) De veg. IV, iii, 4; II, i, 3.

(39) De veg. VI, i, 29.

(40) Cf. por ejemplo, III, i, 2.

(41) De veg. IV, iii, 2.

(42) Id.

(43) De veg. II, ii, 2.

(44) De veg. II, ii, 3.

(45) "Inveniuntur autem duo genera spinarum in plantis. Sunt enim spinae quae ex profundo plantae educuntur, et hae quasi rectae et longae propter calorem materiae. Sunt etiam quaedam spinae, quarum basis non profundatur in corpus plantae, sed stat super corticem extrinsecus adhaerens plantae: et sunt istae spinae breves recurvae anterius et peracutae, sicut sunt spinae tribulorum et bedegar et rosariorum et ramni et aliarum multarum" (De veg. IV, iii, 3).

(46) Id.

(47) De veg. II, i, 4.

(48) Id.

(49) De veg. II, ii, 4.

(50) De veg. II, ii, 5.

(51) Id. II, ii, 4.

(52) De veg. IV, iv, 1.

(53) De veg. II, ii, 4.

(54) De veg. II, ii, 6.

(55) Id.

(56) Id.

(57) Id.

(58) De veg. VI, i, 33.

(59) De veg. II, ii, 7.

(60) De veg. II, ii, 5.

(61) De veg. VI, ii, 9.

(58) "Borago herba est in foliis sicut arnoglossa, et habet folia aspera, et stipitem totum lanuginosum, florem hyacinthinum ut stellam dispositum, in quo est compositio quinque quinariorum, quorum primus est exterior qui fuit theca / floris, et est viridis, secundus autem est hyacinthinus, qui est folia / floris, et postea sunt quinque parvulae eminentiae in flore ipso, et sunt quasi primae extremitates foliorum, et hyacinthinae superius, et inferius albae: post illas ad intrinsecus floris sunt quinque virgulae hyacinthinae longae et rectae, et in medio illarum est una virgula longior eis, et illae sunt spicae floris." (De veg. VI, ii, 3. Lo escrito entre barras falta en la edición de Borgnet.)

(63) De veg. II, ii, 4; II, ii, 5.

(64) De veg. II, ii, 7.

(65) De veg. VI, i, 32.

(66) De veg. II, ii, 6.

(67) Id.

(68) De veg. III, i, 1.

(69) Id. 
(70) De veg. III, i, 2.

(71) De veg. II, ii, 1.

(72) "Primum igitur de fructibus est sciendum, quod nomen fructus plus convenit artis intentioni quam naturae. Fructus enim est illud quo fruitur agricola post laborem agriculturae, et est finis laboris et operis ejus. Secundum autem intentionem naturae semen vocatur, in quo natura intendit conservare et multiplicare speciem propter potentiam generationis completum quam ponit in ipso" (Deveg. III, i, 1). Cfr. et. IV, iii, 2.

(73) De veg. V, i, 3.

(74) De veg. III, i, 2; III, i, 4.

(75) De veg. III, i, 7.

(76) De veg. III, ii, 7; III, i, 5.

(77) De veg. III, i, 5 .

(78) De veg. III, i, 1; III, i, 2.

(79) De veg. III, i, 2.

(80) De veg. III, i, 6.

(81) De veg. III, i, 5.

(82) De veg. III, i, 3; III, i, 4.

(83) Id.

(84) Id.

(85) De veg. III, i, 1.

(86) Id.

(87) De veg. III, i, 5.

(88) De veg. III, i, 1. 